Acta Crystallographica Section D

\section{Biological \\ Crystallography}

ISSN 0907-4449

\section{John E. Ladbury}

Department of Biochemistry and Molecular Biology, University College London,

Gower Street, London WC1E 6BT, England

Correspondence e-mail:

j.ladbury@biochem.ucl.ac.uk

\title{
Measurement of the formation of complexes in tyrosine kinase-mediated signal transduction
}

Isothermal titration calorimetry (ITC) provides highly complementary data to high-resolution structural detail. An overview of the methodology of the technique is provided. Ultimately, the correlation of the thermodynamic parameters determined by ITC with structural perturbation observed on going from the free to the bound state should be possible at an atomic level. Currently, thermodynamic data provide some insight as to potential changes occurring on complex formation. Here, this is demonstrated in the context of in vitro quantification of intracellular tyrosine kinase-mediated signal transduction and the issue of specificity of the important interactions. The apparent lack of specificity in the interactions of domains of proteins involved in early signalling from membrane-bound receptors is demonstrated using data from ITC.

\section{Introduction}

Membrane-localized receptors on the surface of eukaryotic cells receive inputs (e.g. cytokines, growth factors) from the extracellular space. Interaction of these inputs with the receptor initiates an intracellular signal transduction pathway of protein-protein interactions via a conformational change in the receptor and/or a post-translational modification, such as tyrosine phosphorylation, presenting a suitable binding site (Schlessinger, 2000; Hunter, 2000; Pawson \& Nash, 2000; Pawson et al., 2001; Pawson, 2004). A large number of signalling pathways in eukaryotic cells are based on tyrosine kinase (TK) activity. Phosphorylation of tyrosine residues provide targets for downstream protein recognition. These pathways utilize protein-protein interactions which are mediated via homologous domains. For example, Src homology 2 (SH2) and phosphotyrosine-binding (PTB) domains bind directly to sites displaying a phosphotyrosine (pY) moiety. Other domains, such as SH3, WW etc., are additionally found on many of the proteins involved in TKmediated signalling. These pathways are generally assumed to be linear and thus based on a relay of pairwise protein interactions. Since many of these pathways can occur in the same cell and since any given cell can express in excess of 100 proteins with similar domains, a major question as to their ability to maintain mutual exclusivity in cell signalling exists. How, for example, is a given surface on a cognate ligand able to present a site which is sufficiently specific for one $\mathrm{SH} 2$ domain such that it is not also recognized by another similar domain?

To address this question, some definition of specificity is required. Here, a specific protein interaction is thought of as
Received 24 February 2006 Accepted 3 November 2006 
one which will prevail in the context of other competing proteins. Thus, in a typical cell the concentrations of proteins involved in tyrosine signalling pathways are believed to vary by at least two orders of magnitude at the cell surface; thus, for a specific interaction to prevail when nonspecific competitors can be at concentrations that are 100-fold greater, the difference in affinity has to be of the order of at least three orders of magnitude.

To assess the specificity of the interactions between these molecules, the isothermal titration calorimetric method was adopted. This instrument provides accurate determination by direct experiment of affinity in the range of $0.1 \mathrm{~m} M$ to $10 \mathrm{n} M$. In addition, the change in enthalpy $(\Delta H)$ and the change in entropy $(\Delta S)$ for an interaction can be determined, which provide a further level of information on the formation of a complex.

\section{Isothermal titration calorimetry (ITC)}

To address the question above, it is important to quantify the interactions involved in providing the protein-protein signalling pathway. Isothermal titration calorimetry (ITC) provides a rapid method for the measurement of the equilibrium binding constant $\left(K_{\mathrm{B}}=1 / K_{\mathrm{d}}\right)$ of an interaction (Wiseman et al., 1989; Ladbury \& Chowdhry, 1996). The strength of the method, however, as its name suggests, is in the direct determination of the heat associated with going from the unbound to the bound state. The instrument is constructed such that a sample cell (volume $\sim 1.5 \mathrm{ml}$ ) is filled with one component of the interaction. In 'power compensatory' instruments an identical 'reference' cell is housed along with the sample cell in an isothermal jacket (Fig. 1a). The two cells are linked electronically such that they are always maintained at identical temperature (the interaction temperature). The jacket is cooled from the outside at a temperature which is usually 5$10 \mathrm{~K}$ below the required temperature for the experiment. As a result, the reference and sample cells are constantly heated to ensure that they are at the interaction temperature. Thus, there is always an input of power into the cells. Into the sample cell is inserted a syringe which injects accurate volumes of the second component of the interaction. On injection and mixing of the two components a heat effect occurs. If the interaction is exothermic, then to maintain the condition of constant temperature between the two cells less power will be required by the sample cell. Similarly, if the interaction is endothermic more power will be required. The change in power required by the sample cell is therefore measured over time as an injection is made and the contents in the sample cell return to equilibrium. Plotting power against time therefore gives a peak on an equilibrium baseline (Fig. 1b). Integration of this peak with respect to time provides the heat per injection. If the concentrations of the interacting components in the experiment are set up such that the component in the syringe gradually saturates the available binding sites in the cell, then a titration isotherm is obtained (Fig. 1c).

The raw data from the instrument provides a total heat to saturate a known concentration of the component in the cell.
From this the $\Delta H$ is determined. Since this term involves the heats associated with all of the processes which might occur in the interaction (i.e. as well as the heat of binding, there could be effects from events such as protonation, ionization, conformational changes etc.) the subscript 'obs' is used to designate this as an observed $\Delta H$ (e.g. $\left.\Delta H_{\mathrm{obs}}\right)$. The direct determination of the $\Delta H$ is unique to calorimetry and has an advantage over other methods which determine $\Delta H$ indirectly via the van 't Hoff relationship. This advantage is accentuated in systems where there is a large variation of $\Delta H$ with temperature (i.e. a large heat-capacity change) associated with binding since this is not usually incorporated in van 't Hoff calculations. The profile of the binding isotherm allows the concentrations of free and bound ligand to be determined at each point in the titration. From this the $K_{\mathrm{B}}$ can be calculated.

The data from a single experiment can be used to provide a full thermodynamic characterization of the binding event (i.e. the $\Delta H, \Delta G$ and $\Delta S$ ) based on the relationships below. The change in free energy, $\Delta G_{\text {obs }}$ is related to $K_{\mathrm{B}, \mathrm{obs}}$ by

$$
K_{\mathrm{B}, \mathrm{obs}}=\exp \left(-\Delta G_{\mathrm{obs}} / R T\right),
$$

where $R$ is the gas constant and $T$ is the experimental temperature. The change in entropy, $\Delta S_{\text {obs }}$ is related to $\Delta H_{\text {obs }}$ by

$$
\Delta H_{\mathrm{obs}}=\Delta G_{\mathrm{obs}}+T \Delta S_{\mathrm{obs}} .
$$

Conducting the experiment at a range of temperatures provides a way of determining the constant pressure change in heat capacity $\Delta C_{\mathrm{p}, \text { obs }}$ based on the relationship

$$
\Delta C_{\mathrm{p}, \mathrm{obs}}=\mathrm{d} \Delta H_{\mathrm{obs}} / \mathrm{d} T .
$$

The importance of these terms is discussed and a tutorial on the ITC methodology is presented in a book published in 2004 on the subject of applications of biocalorimetry (Thomson \& Ladbury, 2004).

As well as providing a full thermodynamic characterization of a molecular interaction, the ITC experiment also allows the determination of the stoichiometry. For a simple one-to-one interaction the profile of the ITC isotherm is sigmoidal (Fig. 1c). The stoichiometry can readily be seen as the molar ratio at the equivalence point of the titration. In a situation where there are two identical binding sites on the component in the calorimeter cell for the component in the syringe, the equivalence point of the sigmoidal isotherm will be at a molar ratio of two. In the opposite case where there are two binding sites on the component in the syringe, the mole ratio at equivalence will be 0.5 . In the case where the binding sites are not identical, they will have a different affinity and possibly different $\Delta H_{\text {obs. }}$. The isotherm obtained in these situations initially shows the tightest binding event gradually saturating and any free ligand binding to the weaker site. If the affinities of the individual interactions are sufficiently different, the sigmoidal shape is no longer found and depending on the respective enthalpies of the binding events a clear step function is observed in the isotherm. The stoichiometry of these more complex systems can also be determined by fitting for a model including each event. 


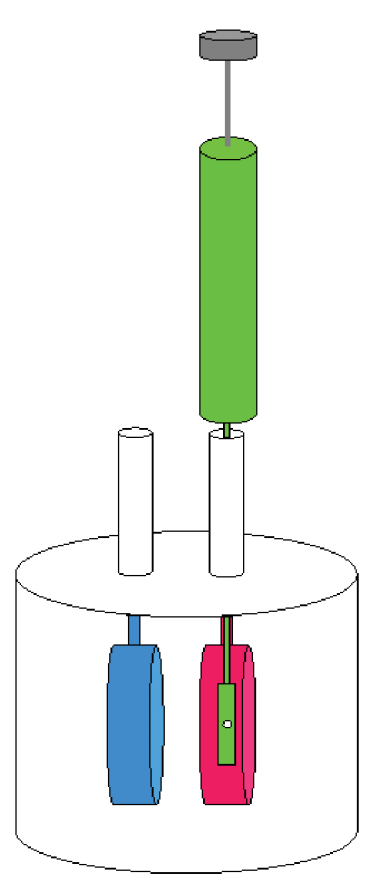

(a)

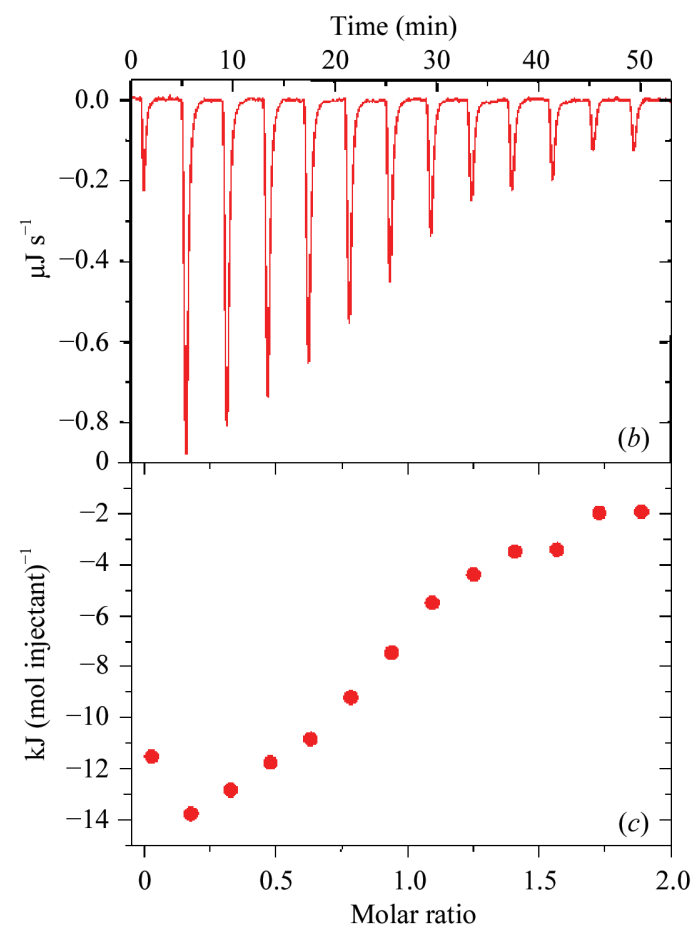

Figure 1

(a) Schematic of an isothermal titration calorimeter. (b) Raw data output and (c) integrated data (a) shows the sample cell and the reference cell in an isothermal jacket. The syringe is inserted in the sample cell and a series of injections are made. The injection of one component into the other results in an exothermic heat effect. After each injection, once the interaction is complete the power returns to an equilibrium value $(b)$. The concentration regime between the two components is set up such that after a series of injections the binding sites available in the sample cell are gradually saturated. The profile of the binding isotherm $(c)$ is fitted to obtain the thermodynamic parameters (see text).

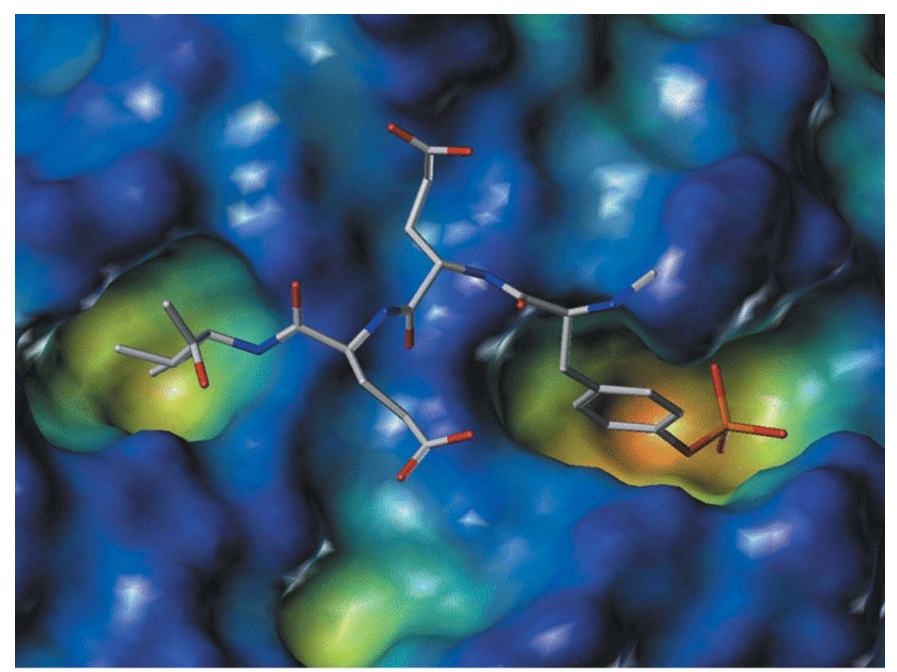

Figure 2

Surface representation of the binding site of the Src SH2 domain showing the 'two-pinned plug' mode of binding. The colours are used to emphasize depth of pocket only.

The ITC experiment therefore provides not only the affinity and hence the $\Delta G$ of an interaction, but also a further level of quantification, i.e. the $\Delta H$ and $\Delta S$. The value of these additional terms is largely in the interpretation of a binding event at the atomic level and thus high-resolution structural detail is required. Since the $\Delta H$ term is proportional to the net change in number or strengths of noncovalent bonds on going from the free to the bound state, if structural information of the apo form and the complex of the biomolecules involved is available then a correlation between these and thermodynamics can be observed or predicted. This is perhaps most useful in comparing one ligand with another and demonstrating how structural perturbation (change in noncovalent bonds, removal of water molecules, burial of surface area etc.) on going from one complex to another results in a thermodynamic effect.

\section{Determination of the specificity of $\mathrm{SH2}$-domain interactions in tyrosine kinase-mediated signal transduction}

As stated above, the protein-protein interactions, at least in the early stages of a TK-mediated signal transduction event, are often based on the binding of independent domains. ITC has been used to measure the interactions of several examples of these domains to assess the affinity and hence the level of specificity existing in these interactions.

The interactions of $\mathrm{SH} 2$ domains provide the primary recognition site in tyrosine kinase-mediated signalling since they recognize protein ligands with a phosphorylated tyrosine residue (pY). The specificity for interactions of $\mathrm{SH} 2$ domains has been reported and is widely considered to be based on recognition of a short series of amino acids usually C-terminal and proximal to the pY (Songyang et al., 1993). The structural basis for this was demonstrated in a number of studies which revealed that the pY residue delved into a deep positively charged pocket, whilst the residue which was three residues C-terminal of the pY (pY +3) bound in another pocket (Fig. 2; see also Kuriyan \& Cowburn, 1993, 1997). These pockets were believed to provide the basis for recognition on the binding surface. Interactions of SH2 domains have been widely characterized both from a structural and a thermodynamic perspective. In this work, the $\mathrm{SH} 2$ domain from the protein Src was adopted as a model system. This $\mathrm{SH} 2$ domain was shown from screening studies to bind 'specifically' to the sequence pYEEI (Songyang et al., 1993). High-resolution structural data show how the interaction of a peptide containing this motif is based around the two deep pockets described above. The interaction is described as resembling a 'two-pinned plug' (Waksman et al., 1992, 1993; Bradshaw et al., 1998) with the pY and the $\mathrm{pY}+3$ isoleucine forming the pins (Fig. 2). It should be pointed out that since all $\mathrm{SH} 2$ domains require the interaction of the pY moiety on the cognate ligand, this provides no specificity. 
Table 1

Binding of tyrosyl phosphopeptides to the Src SH2 domain at $298 \mathrm{~K}$.

\begin{tabular}{|c|c|c|c|c|c|}
\hline Peptide & $\begin{array}{l}K_{\mathrm{d}} \\
(\mu M)\end{array}$ & $\begin{array}{l}\Delta H_{\text {obs }} \\
\left(\mathrm{kJ} \mathrm{mol}^{-1}\right)\end{array}$ & $\begin{array}{l}T \Delta S_{\text {obs }} \\
\left(\mathrm{kJ} \mathrm{mol}^{-1}\right)\end{array}$ & $\begin{array}{l}\Delta G_{\text {obs }} \\
\left(\mathrm{kJ} \mathrm{mol}^{-1}\right)\end{array}$ & Reference $\dagger$ \\
\hline \multicolumn{6}{|c|}{ Peptides based on 'specific' sequence } \\
\hline KGGQpYEEIPIP & 0.55 & -35.4 & 0.5 & -36.0 & $a$ \\
\hline KGGQpYEEIPIP & 0.77 & -33.4 & 1.7 & -35.1 & $a$ \\
\hline EPQpYEEIPIYL & 0.09 & -38.7 & 1.4 & -40.1 & $b$ \\
\hline PQpYEEIPI & 0.18 & -32.3 & 6.3 & -38.6 & $c$ \\
\hline PQpYEEIPI & 0.25 & -31.5 & 6.3 & -37.8 & $d$ \\
\hline PQpYEEIPI & 0.27 & -31.5 & 6.3 & -37.8 & $e$ \\
\hline PQpYEEIPI & 0.2 & -27.3 & 10.9 & -38.2 & $f$ \\
\hline $\mathrm{pY}$ & 175 & 3.3 & 24.1 & -21.4 & \\
\hline \multicolumn{6}{|c|}{ Peptides with substitution in $\mathrm{pY}+1$ position of 'specific' sequence } \\
\hline PQpYQEIPI & 0.47 & -31.9 & 4.2 & -36.1 & $g$ \\
\hline PQpYDEIPI & 0.18 & -37.4 & 1.3 & -38.6 & $g$ \\
\hline PQpYAEIPI & 0.34 & -32.3 & 4.2 & -36.5 & $g$ \\
\hline PQpYGEIPI & 6.25 & -20.2 & 9.7 & -29.8 & $g$ \\
\hline PQpYAEIPI & 0.35 & -32.3 & 4.2 & -36.5 & $e$ \\
\hline \multicolumn{6}{|c|}{ Peptides with substitution in $\mathrm{pY}+2$ position of 'specific' sequence } \\
\hline PQpYEYIPI & 0.66 & -26.8 & 8.4 & -35.2 & $d$ \\
\hline PQpYEQIPI & 0.53 & -31.9 & 4.2 & -36.1 & $c$ \\
\hline PQpYEDIPI & 0.42 & -26.0 & 10.5 & -36.5 & $c$ \\
\hline PQpYEAIPI & 1.04 & -26.5 & 8.0 & -34.4 & $c$ \\
\hline PQpYEAIPI & 1.0 & -26.46 & 7.98 & -34.44 & $e$ \\
\hline PQpYEGIPI & 1.96 & -25.2 & 7.6 & -32.8 & $c$ \\
\hline \multicolumn{6}{|c|}{ Peptides with substitution in $\mathrm{pY}+3$ position of 'specific' sequence } \\
\hline EPQpYEEVPIYL & 0.16 & -28.6 & 10.2 & -38.8 & $b$ \\
\hline EPQpYEEEPIYL & 0.21 & -32.7 & 5.4 & -38.1 & $b$ \\
\hline EPQpYEEWPIYL & 0.31 & -32.2 & 4.9 & -37.1 & $h$ \\
\hline EPQpYEEDPIYL & 0.38 & -27.5 & 9.1 & -36.6 & $h$ \\
\hline PQpYEELPI & 0.43 & -23.5 & 13.0 & -36.5 & $c$ \\
\hline PQpYEEVPI & 0.46 & -22.7 & 13.9 & -36.5 & $c$ \\
\hline PQpYEEAPI & 1.75 & -21.4 & 11.3 & -32.7 & $c$ \\
\hline PQpYEEGPI & 0.39 & -15.1 & 16.0 & -31.1 & $c$ \\
\hline \multicolumn{6}{|c|}{ Completely randomized sequences } \\
\hline PQpYQPGEN & 29.4 & -19.3 & 6.7 & -26.0 & $f$ \\
\hline EPQpYQPGEN & 14.3 & -25.7 & 2.0 & -27.7 & $b$ \\
\hline
\end{tabular}

$\dagger$ References for the ITC data: a, Ladbury et al. (1995); b. Chung et al. (1998); c, Bradshaw \& Waksman (1999); $d$, Lubman \& Waksman (2003); $e$, Lubman \& Waksman (2002); f, Bradshaw et al. (1998); g, Bradshaw et al. (1999); $h$, Henriques \& Ladbury (2001).

ITC was used to assess the contribution of the pY moiety to the total change in free energy of binding of a ligand. Since the interaction of this molecule is inherently weak, competition assays were used to determine the thermodynamic parameters for the interaction (Table 1). The $\mathrm{pY}$ binds to the $\mathrm{SH} 2$ domain at $298 \mathrm{~K}$ with a $K_{\mathrm{B}}$ of $6 \times 10^{3} M^{-1}$ and hence a $\Delta G_{\mathrm{obs}}=$ $-21.4 \mathrm{~kJ} \mathrm{~mol}^{-1}$. As described below, this represents a significant proportion of the total $\Delta G_{\mathrm{obs}}$ of cognate ligand binding.

Since the specificity of the interaction is derived from the amino acids adjacent to and C-terminal of the pY residue, binding data were obtained using ITC for the interaction of a series of peptides in which the sequence of amino acids in these positions were systematically randomized. Remarkably, the data (Table 1) reveal that there is really very little specificity in the interaction of the C-terminal domain itself. In fact, all of the different peptide sequences bind with at most only a 60 -fold difference in $K_{\mathrm{d}}$, between approximately 0.1 and $6 \mu M$. This can be exemplified in looking at the amino acids in the pY +3 position. In a given series of peptides, changing the amino acid from the 'specific' isoleucine residue to anything from a small acidic group (Asp) to a large aromatic side chain (Trp) has at most a fourfold effect on binding. This is clearly not showing the levels of affinity conducive to specific binding. In fact, this seems to represent a highly promiscuous binding site. When the entire sequence proximal to the $\mathrm{pY}$ is randomized (i.e. pYQPG) the affinity is reduced, but only results in a maximum of about a 160-fold difference for equivalent peptides (Chung et al., 1998; Henriques \& Ladbury, 2001).

The determination of the $\Delta H_{\mathrm{obs}}$ and $\Delta S_{\mathrm{obs}}$ terms for the interactions allows some discrimination between the binding events to be highlighted. For example, all of the interactions have a favourable entropic contribution. Generally, this entropic effect is maximized with changes in the $\mathrm{pY}+2$ and $\mathrm{pY}+3$ positions. In the case of the $\mathrm{pY}+2$ this is likely to be the effect of the release of a group of water molecules which are hydrogen bonded into the complex interface as seen in the structure of the specific peptide complex (Waksman et al., 1993; Henriques \& Ladbury, 2001). In the case of the pY +3 position, the increased favourable entropic term is likely to result from the peptide not being able to make a strong 'twopinned plug' interaction and thus being more dynamic in the complex form (Ladbury et al., 1996). In almost all cases the $\Delta G_{\text {obs }}$ term is little affected owing to an enthalpic compensatory effect.

This same lack of specificity can be observed in other independent domain interactions. For example, PTB domains appear to offer limited specificity in their direct interactions with pY-containing ligands (Ladbury, 2005) and the majority of SH3 domains studied show small differences in binding different proline-rich motifs (Ladbury \& Arold, 2000; O'Rourke \& Ladbury, 2003).

\section{Is specificity imposed by the simultaneous binding of more than one domain?}

The data derived from ITC on the binding of peptides to single domains suggest that there is a lack of specificity imposed by

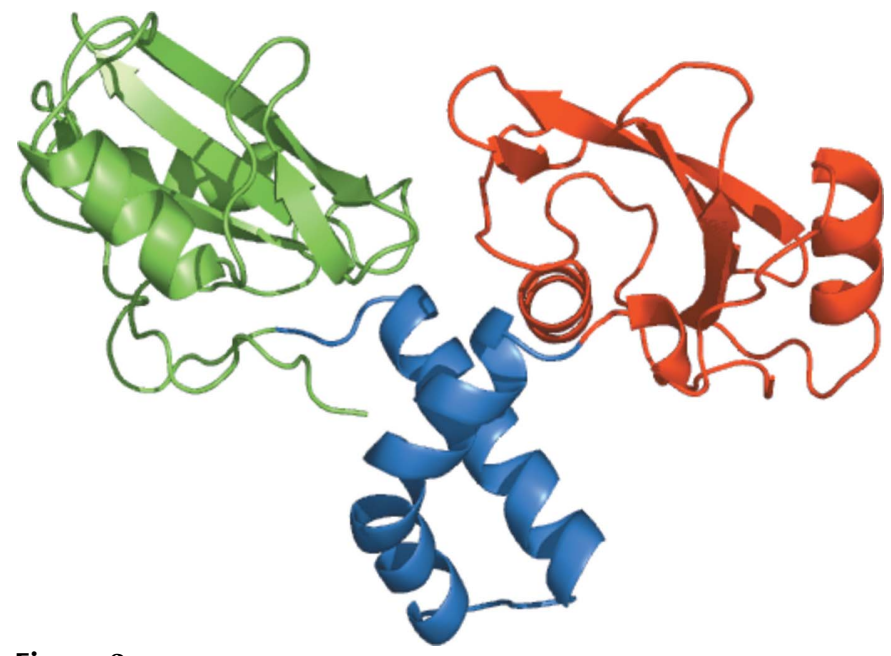

Figure 3

Ribbon diagram representation of the apo-ZAP70 SH2 domains and inter-SH2 domain linker (based on Folmer et al., 2002). The N-terminal $\mathrm{SH} 2$ domain is shown in green and the C-terminal SH2 domain is shown in red. These SH2 domains have to come together to form the binding site for the ITAM bisphosphorylated peptide (see Hatada et al., 1995). 
Table 2

Binding of peptides to the SH2 and SH3 domains of Fyn at $298 \mathrm{~K}$.

\begin{tabular}{lrlll}
\hline Peptide & $\begin{array}{l}K_{\mathrm{d}} \\
(\mu M)\end{array}$ & $\begin{array}{l}\Delta H_{\mathrm{obs}} \\
\left(\mathrm{kJ} \mathrm{mol}^{-1}\right)\end{array}$ & $\begin{array}{l}T \Delta S_{\text {obs }} \\
\left(\mathrm{kJ} \mathrm{mol}^{-1}\right)\end{array}$ & $\begin{array}{l}\Delta G_{\text {obs }} \\
\left(\mathrm{kJ} \mathrm{mol}^{-1}\right)\end{array}$ \\
\hline pYEEI & 2.5 & -45 & -13 & -32 \\
PXXP & 62.0 & -68 & -43 & -25 \\
Sum of thermodynamic parameters & & $-\mathbf{1 1 3}$ & & $-\mathbf{5 7}$ \\
FAK peptide & 0.19 & -132 & -94 & -38
\end{tabular}

these interactions (Ladbury \& Arold, 2000). Many proteins involved in TK-mediated signalling have more than one domain. It has been suggested that to increase specificity, the binding of two domains could be important. For example, the binding of two domains to a single cognate ligand will potentially increase the affinity owing to the cooperative effect of the individual binding sites on the domains. This effect should be manifested in a $\Delta G$ term which is more favourable than the additive $\Delta G$ terms for the interactions of the individual domains. Furthermore, a selective advantage could be incurred by the fact that the two domains will have a distinct relative positioning with respect to one another, which will require the correct juxtaposition of their binding sites on the cognate protein. ITC has been used to measure the binding of combined domains to peptide ligands bearing the appropriate binding sites. In most cases, no significant increase in affinity has been observed.

A good example of this observation is from a study of the binding of the Fyn protein (an Src-family tyrosine kinase) to focal adhesion kinase (FAK; Arold et al., 2001). Fyn binds to FAK through interactions of its $\mathrm{SH} 2$ and $\mathrm{SH} 3$ domains. The domains recognize tyrosyl-phosphorylated and proline-rich sites on the FAK protein, respectively. ITC was used to determine the thermodynamic parameters associated with the binding of the $\mathrm{SH} 2$ and $\mathrm{SH} 3$ to their cognate ligands in isolation and also of the binding of the two domains to a peptide containing both recognition motifs. The level of specificity for the binding of peptides with the individual recognition motifs to the individual domains (Table 2) could be compared with those from the binding of the intact FAK peptide. As can be seen, the $\mathrm{SH} 2$ domain binds to a phosphotyrosyl peptide and the $\mathrm{SH} 3$ domain binds to a proline-rich sequence with $K_{\mathrm{d}}$ values in the micromolar range. The binding of the peptide from FAK only bound approximately an order of magnitude tighter than the SH2-domain pY-peptide interaction. Accordingly, the $\Delta G_{\mathrm{obs}}$ for the binding of the FAK peptide to the polypeptide containing the pair of domains is only slightly larger than that for the SH2-domain interaction alone and thus shows no cooperative effect. The $\Delta H_{\text {obs }}$ and $\Delta S_{\text {obs }}$ terms derived from the ITC experiments reveal that the failure to produce the expected greater than additive $\Delta G_{\text {obs }}$ value is largely the result of a highly unfavourable entropic effect. Thus, although the interaction of the FAK peptide increases the net number/strength of noncovalent interactions in the complex, there is a sizeable entropic penalty. This is hypothesized to be a consequence of a conformational change which is required to align the binding
Table 3

Binding of peptides to the SH2 domain of ZAP70 at $298 \mathrm{~K}$.

\begin{tabular}{lclll}
\hline Peptide & $\begin{array}{l}K_{\mathrm{d}} \\
(\mu M)\end{array}$ & $\begin{array}{l}\Delta H_{\mathrm{obs}} \\
\left(\mathrm{kJ} \mathrm{mol}^{-1}\right)\end{array}$ & $\begin{array}{l}T \Delta S_{\mathrm{obs}} \\
\left(\mathrm{kJ} \mathrm{mol}^{-1}\right)\end{array}$ & $\begin{array}{l}\Delta G_{\mathrm{obs}} \\
\left(\mathrm{kJ} \mathrm{mol}^{-1}\right)\end{array}$ \\
\hline $\begin{array}{l}\text { N-terminal pY peptide } \\
\begin{array}{l}\text { C-terminal pY peptide } \\
\text { Sum of thermodynamic }\end{array}\end{array}$ & 142 & -48 & -26 & -22 \\
$\begin{array}{l}\text { parameters } \\
\text { Bisphosphopeptide }\end{array}$ & 0.033 & -47 & -27 & -20 \\
\hline
\end{tabular}

sites on the peptide to enable docking with the $\mathrm{SH} 2-\mathrm{SH} 3$ domain-containing polypeptide.

Binding data from other pairwise interactions of domains have also failed to produce the expected additive $\Delta G$. This suggests that the interactions, like those of the individual domains, have not been optimized to be of high affinity and thus have limited specificity.

One multidomain binding system in which some enhancement in affinity has been obtained is the binding of the $\mathrm{SH} 2$ domains of ZAP70 to a ligand corresponding to one of the subunits of the T-cell receptor (O'Brien et al., 2000). The $\zeta$-chain activating protein ZAP70 plays a role in T-cell activation. It possesses two SH2 domains separated by 65 residues (Fig. 3) which bind to the bisphosphorylated immunoreceptor tyrosine-based activation motif (ITAM) on the $\zeta$ subunit of the CD3 T-cell receptor. Activated ITAM motifs have a consensus amino-acid sequence $\mathrm{pY} X X(\mathrm{~L} / \mathrm{I})-X_{7 / 8}-\mathrm{pY} X X_{9}(\mathrm{~L} / \mathrm{I})$. Each pY moiety binds to one SH2 domain. Peptides based on the monophosphorylated ITAMs using ITC revealed weak binding to ZAP70 $\left(K_{\mathrm{d}} \simeq 0.1 \mathrm{~m} M\right.$; see Table 3; O'Brien et al., 2000). The bisphosphorylated peptide bound with a stoichiometry of unity and an affinity which was nearly four orders of magnitude tighter than the monophosphorylated form (32 $\mathrm{nM}$ ). However, considering this value for the double $\mathrm{SH} 2$ binding event compared with the binding data for the single $\mathrm{SH} 2$ domains reported in Tables 1 and 2 , this is only approximately an order of magnitude stronger than the interaction of a single $\mathrm{SH} 2$ domain. The apparent lack of a significant cooperative effect of the $\Delta G_{\text {obs }}$ values again suggests that the interaction has not evolved to optimize affinity (Table 3).

The thermodynamic analysis in Table 3 reveals that the additive nature of the $\Delta G_{\text {obs }}$ term and hence the lack of high affinity for the overall binding effect is based on the weak interactions of the $\mathrm{SH} 2$ domains in isolation and the apparent lack of additivity in the favourable $\Delta H_{\mathrm{obs}}$. It is interesting to note that the $\Delta S_{\text {obs }}$ term for the bispeptide interaction is more favourable than that for the individual $\mathrm{SH} 2$ interactions. This suggests that an effect such as liberation of water into bulk solvent from a surface occurs. This expected burial of surface could result from a conformational change in ZAP70 which accompanies binding. The crystal structure of the ZAP70ITAM complex reveals that ZAP70 is unique amongst the tyrosine kinase proteins in that the $\mathrm{N}$-terminal pY pocket involves extensive interactions between the two SH2 domains (Fig. 2; Hatada et al., 1995; Graef et al., 1997; Ottinger et al., 1998; Folmer et al., 2002). This pocket appears to form when 
the ITAM binds. This requires a large structural perturbation. Furthermore, it is known that for the tandem domain interaction to occur the linker region between the $\mathrm{SH} 2$ domains also has to undergo a significant conformational change to allow the formation of the $\mathrm{N}$-terminal $\mathrm{SH} 2$ domain. This linker region folds into a coiled-coil structure. Consequently, a significant burial of surface occurs which is likely to release water and provide the entropic effect observed in this case.

\section{Implications for tyrosine kinase-mediated signal transduction}

The lack of the expected high levels of specificity between the interacting proteins in TK-mediated signalling suggests that the dogma of linear processing has to be reconsidered. If this form of signalling was based simply on protein-protein recognition in bimolecular relays then 'crossed lines' amongst different pathways would be expected and hence inappropriate signals would be transduced. Clearly, this is not the case, so another mechanism for maintaining mutual exclusivity must be operating. Currently, this is hypothesized to be, at least in early signalling, based on the formation of protein complexes which require multiple interactions. Thus, a signal is only transduced when the appropriate proteins in the correct order and stoichiometry are assembled (Ahmed et al., 2005; O'Rourke \& Ladbury, 2003)

\section{Summary}

It is becoming increasingly clear that the combination of the complementary information provided by high-resolution structural determination and thermodynamic data from ITC is the only way to understand fully the formation of a biomolecular complex. In principle, ITC can be used to measure any noncovalent interaction since the transfer of heat either exothermically or endothermically is a ubiquitous property of noncovalent bond formation/breaking. Thus, the correlation of high-resolution structural detail with data derived from ITC experiments provides a very powerful analysis of a binding event. Ultimately, the energetics of the interaction in terms of the formation/breaking of the bonds seen in the apo and complex structures will provide a unique insight to biomolecular interactions and enable effective ligand design/ optimization in therapeutic applications.

The value of these thermodynamic terms has not been exploited fully in providing a biological description of an interaction or in drug development. This is largely owing to the lack of data available to compare between systems. Thus, as more data are published a greater potential for correlating structural and thermodynamic parameters will emerge. An attempt to collect and make structural and thermodynamic data available has recently been provided by the SCORPIO database (http://www.biochem.ucl.ac.uk/scorpio/ scorpio.html).

\section{References}

Ahmed, Z., Beeton, C. A., Williams, M. A., Clements, D., Baldari, C. T. \& Ladbury, J. E. (2005). J. Mol. Biol. 353, 1001-1010.

Arold, S. T., Ulmer, T. S., Mulhern, T. D., Werner, J. M., Ladbury, J. E., Campbell, I. D. \& Noble, M. E. M. (2001). J. Biol. Chem. 276, 17199-17205.

Bradshaw, J. M., Grucza, R. A., Ladbury, J. E. \& Waksman, G. (1998). Biochemistry, 37, 9083-9090.

Bradshaw, J. M., Mitaxov, V. \& Waksman, G. (1999). J. Mol. Biol. 293, 971-985.

Bradshaw, J. M. \& Waksman, G. (1999). Biochemistry, 38, 5147-5154.

Chung, E., Henriques, D., Renzoni, D., Zvelebil, M., Bradshaw, J. M., Waksman, G., Robinson, C. V. \& Ladbury, J. E. (1998). Structure Fold. Des. 6, 1141-1151.

Folmer, R. H. A., Geschwindner, S. \& Xue, Y. F. (2002). Biochemistry, 41, 14176-14184.

Graef, I. A., Holsinger, L. J., Diver, S., Schreiber, S. L. \& Crabtree, G. R. (1997). EMBO J. 16, 5618-5628.

Hatada, M. H., Lu, X., Laird, E. R., Green, J., Morgenstern, J. P., Lou, M., Marr, C. S., Phillips, T. B., Ram, M. K., Theriault, K., Zoller, M. J. \& Karas, J. L. (1995). Nature (London), 377, 32-38.

Henriques, D. A. \& Ladbury, J. E. (2001). Arch. Biochem. Biophys. 390, $158-168$.

Hunter, T. (2000). Cell, 100, 113-127.

Kuriyan, J. \& Cowburn, D. (1993). Curr. Opin. Struct. Biol. 3, 828-837.

Kuriyan, J. \& Cowburn, D. (1997). Annu. Rev. Biophys. Biomol. Struct. 26, 259-288.

Ladbury, J. E. (2005). Protein Reviews, Vol. 3, Proteomics and Protein-Protein Interactions: Biology, Chemistry, Bioinformatics and Drug Design, edited by G. Waksman, pp. 165-184. New York: Kluwer/Plenum.

Ladbury, J. E. \& Arold, S. (2000). Chem. Biol. 7, R3-R8.

Ladbury, J. E. \& Chowdhry, B. Z. (1996). Chem. Biol. 3, 791-801.

Ladbury, J. E., Hensman, M., Panayotou, G. \& Campbell, I. D. (1996). Biochemistry, 35, 11062-11069.

Ladbury, J. E., Lemmon, M. A., Zhou, M., Green, J., Botfield, M. C. \& Schlessinger, J. (1995). Proc. Natl Acad. Sci. USA, 92, 3199-3203.

Lubman, O. Y. \& Waksman, G. (2002). J. Mol. Biol. 316, 91-304.

Lubman, O. Y. \& Waksman, G. (2003). J. Mol. Biol. 328, 55-668.

O'Brien, R., Rugman, P., Renzoni, D., Layton, M., Handa, R., Hilyard, K., Waterfield, M. D., Driscoll, P. C. \& Ladbury, J. E. (2000). Protein Sci. 9, 570-579.

O'Rourke, L. \& Ladbury, J. E. (2003). Acc. Chem. Res. 36, 410-416.

Ottinger, E. A., Botfield, M. C. \& Shoelson, S. E. (1998). J. Biol. Chem. 273, 729-735.

Pawson, T. (2004). Cell, 116, 191-203.

Pawson, T., Gish, G. D. \& Nash, P. (2001). Trends Cell Biol. 11, 504-511.

Pawson, T. \& Nash, P. (2000). Genes Dev. 14, 1027-1047.

Schlessinger, J. (2000). Cell, 103, 211-225.

Songyang, Z., Shoelson, S. E., Chaudhuri, M., Gish, G., Pawson, T., Haser, W. G., King, F., Roberts, T., Ratnofsky, S., Lechleider, R. J., Neel, B. G., Birge, R. B., Fajardo, J. E., Chou, M. M., Hanafusa, H., Schaffhausen, B. \& Cantley, L. C. (1993). Cell, 72, 767-778.

Thomson, J. A. \& Ladbury, J. E. (2004). Biocalorimetry 2: Applications of Calorimetry in the Biological Sciences, edited by J. E. Ladbury \& M. L. Doyle, pp. 37-58. Chichester: John Wiley \& Sons.

Waksman, G., Kominos, D., Robertson, S. C., Pant, N., Baltimore, D., Birge, R. B., Cowburn, D., Hanafusa, H., Mayer, B. J., Overduin, M., Resh, M. D., Rios, C. B., Silverman, L. \& Kuriyan, J. (1992). Nature (London), 358, 646-653.

Waksman, G., Shoelson, S. E., Pant, N., Cowburn, D. \& Kuriyan, J. (1993). Cell, 72, 779-790.

Wiseman, T., Williston, S., Brandts, J. F. \& Lin, L.-N. (1989). Anal. Biochem. 179, 131-137. 